\title{
Secrecy Performance Analysis for Reconfigurable Intelligent Surface aided NOMA Network
}

\author{
Zhiqing Tang*, Tianwei Hou ${ }^{\dagger}$, Yuanwei Liu ${ }^{\ddagger}$, and Jiankang Zhang*§ \\ * School of Information Engineering, Zhengzhou University, Zhengzhou, CN \\ $\dagger$ School of Electronic and Information Engineering, Beijing Jiaotong University, Beijing, CN \\ $\ddagger$ School of Electronic Engineering and Computer Science, Queen Mary University of London, London, UK \\ $\S$ Department of Computing \& Informatics, Bournemouth University, Bournemouth, UK
}

\begin{abstract}
Reconfigurable Intelligent Surface (RIS) technology is emerging as a promising performance enhancement for nextgeneration wireless networks in terms of the quality of service and radio connectivity. Inspired by the promising potential of RIS technology, we investigate the secrecy performance of the downlink RIS-aided non-orthogonal multiple access network. To characterize the network's performance, the expectation of the new channel statistics for the reflected links with Nakagami- $m$ fading is derived. Furthermore, the performance of the proposed network is evaluated according to the secrecy outage probability (SOP). The closed-form expressions of the SOP are derived. To obtain further insights, the asymptotic SOP and secrecy diversity orders are obtained. Our analytical results demonstrate that: 1) the expectation of channel gain for the reflected links is determined by the number of RISs and the Nakagami- $m$ fading parameters; 2) Both the SOP of user Bob1 and the SOP of user Bob2 are 1 when the number of RISs is sufficiently large; 3) The secrecy diversity orders are affected by the number of RISs and Nakagami- $m$ fading parameters.
\end{abstract}

\section{INTRODUCTION}

In recent years, Reconfigurable Intelligent Surface (RIS) has been proposed as a new technology to deal with the randomness and uncontrollability of wireless signal propagation [1]. RIS has the ability to overcome the negative effects of natural wireless propagation by controlling the scattering, reflection, and refraction characteristics of the radio waves [2,3]. Also, RIS provides a new direction for the design and optimization of wireless communication networks. By appropriately adjusting the amplitude-reflection and phase coefficients, the RISs can enhance the received signals $[4,5]$, or eliminate the undesired signals such as co-channel interference $[6,7]$.

Power domain non-orthogonal multiple access $\left(\mathrm{NOMA}^{1}\right.$ ) has the ability to provide services to multiple users in the same physical resource block (e.g., time and frequency) at the same time, thereby significantly improving SE and connection density $[8,9]$. As demonstrated in [10], to unleash the full potential of NOMA is important to ensure that an appropriate power difference exists between the users. The RIS has the ability to change the channel gains, which is able to enhance the performance of NOMA by arousing desirable differences of channel gains among the users.

\footnotetext{
${ }^{1}$ Throughout this paper, we focus our attention on the family of powerdomain NOMA. We simply use "NOMA" to refer to "power-domain NOMA" in the following.
}

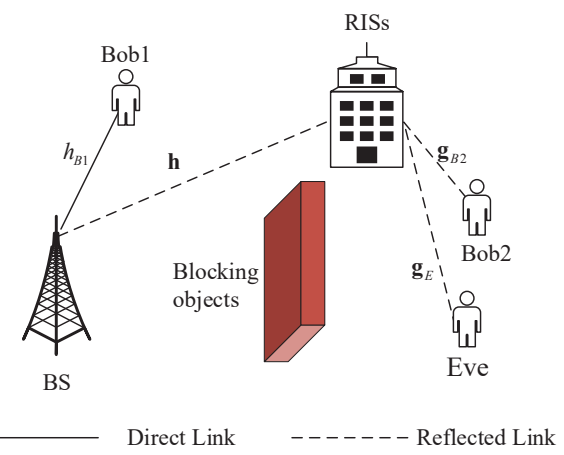

Fig. 1: System model

Motivated by the potential joint benefits of RIS and NOMA, the RIS-aided NOMA networks have been investigated recently [11-13]. Considering the broadcast nature of wireless transmission, the issue of physical layer security (PLS) attracted widespread interests. Although rigorous efforts have been done in PLS of wireless communications, overall progress has been relatively slow [14]. However, the emergence of RIS technology provides a new solution for PLS problem. In [15], the authors studied the secrecy performance of an RIS-aided wireless communication network in the presence of an eavesdropper (Eve). Similarly, in [16], the authors investigated an RIS-aided secure wireless communication network, where the eavesdropping channels are stronger than the legitimate communication channels. In [17], the authors investigated whether the use of artificial noise is helpful to enhance the secrecy rate in the RIS-aided network. Most of the existing works on PLS in the RIS-aided networks studied the optimization problem to maximize secrecy rate [18-20]. As mentioned above, PLS has been studied in various scenarios, but it is rarely studied in RIS-aided NOMA, which motivates this contribution.

\section{System Model}

As show in Fig. 1, we consider the secure downlink (DL) of an IRS-aided NOMA network, where a BS communicates with two legitimate users (LUs) in the presence of an Eve. It is assumed that the BS, LUs and Eve are equipped with a single antenna. For the two LUs, the NOMA transmission protocol is invoked. We also assumed that Eve has powerful detection capability which is capable of overhearing the messages of the 
LUs. We have $\mathrm{N}$ intelligent surfaces at the appropriate location. More specifically, user Bob2 is the cell-edge user which needs help from the RIS to communicate with the BS. At the same time, user Bob1 is the cell-center user that can communicate with the BS directly. Besides, there is no direct link between the RIS and user Bob1, as well as that between the BS with user Bob2 and Eve, due to long-distance and blocking objects.

The small-scale fading vector between the BS and RISs is denoted by

$$
\mathbf{h}=\left[h_{1}, h_{2}, \cdots, h_{N}\right]^{T},
$$

The small-scale fading vectors between RISs and user Bob2 and that between RISs and Eve are given by

$$
\mathbf{g}_{B 2}=\left[g_{2,1}, g_{2,2}, \cdots, g_{2, N}\right],
$$

and

$$
\mathbf{g}_{E}=\left[g_{E, 1}, g_{E, 2}, \cdots, g_{E, N}\right],
$$

respectively. The elements in $\mathbf{h}, \mathbf{g}_{B 2}$ and $\mathbf{g}_{E}$ follow the Nakagami- $m$ distribution with fading parameters $m_{1}, m_{2}$, and $m_{3}$, respectively.

The BS sends $s=\sqrt{a_{1}} s_{1}+\sqrt{a_{2}} s_{2}$ to LUs with the power of $P$. where $s_{1}$ and $s_{2}$ are the signal intended for user Bob1 and user Bob2, respectively. $\sqrt{a_{1}}$ and $\sqrt{a_{2}}$ are the power allocation factors of user Bob1 and user Bob2, respectively. $d_{B 1}$ and $d_{1}$ denote the distances from the BS to user Bob1 and the RIS, respectively, $d_{B 2}$ denotes the distance from the RIS to user Bob2, $\alpha_{B 1}, \alpha_{1}$ and $\alpha_{B 2}$ denote the path loss exponents for BS-Bob1 link, BS-RIS links and RIS-Bob2 links, respectively.

The signal received by user Bob1, user Bob2 and Eve are given by

$$
\begin{gathered}
y_{1}=h_{B 1} \sqrt{d_{B 1}^{-\alpha_{B 1}}} P s+n_{1}, \\
y_{2}=\mathbf{g}_{B 2} \mathbf{\Phi} \mathbf{h} \sqrt{d_{1}^{-\alpha_{1}} d_{B 2}^{-\alpha_{B 2}}} P s+n_{2},
\end{gathered}
$$

and

$$
y_{E}=\mathbf{g}_{E} \boldsymbol{\Phi} \mathbf{h} \sqrt{d_{1}^{-\alpha_{1}} d_{E}^{-\alpha_{E}}} P s+n_{E},
$$

respectively, where $n_{1}$ and $n_{2}$ and $n_{E}$ denote additive white Gaussian noises (AWGNs) with variance $\sigma^{2}$. In addition, $\boldsymbol{\Phi} \triangleq \operatorname{diag}\left[\beta_{1} \phi_{1}, \beta_{2} \phi_{2}, \ldots, \beta_{N} \phi_{N}\right]$ is a diagonal matrix, which represents the effective phase shift applied by all intelligent surfaces. $\beta_{n} \in(0,1]$ denotes the amplitude reflection coefficient of RISs, while $\phi_{n}=\exp \left(j \theta_{n}\right), j=\sqrt{-1}, \forall n=1,2, \ldots, N$, and $\theta_{n} \in[0,2 \pi)$ represents the phase shift caused by the $n$-th intelligent surface.

\section{Secrecy Performance Analysis}

In this section, we consider the RIS design as in [12]. Specifically, in order to simultaneously control multiple RISs, the channel state information (CSI) of the paired NOMA users channels is assumed to be perfectly available. However, the CSI of Eve is not available.

\section{A. New Channel Statistics}

According to the previous assumption, the instantaneous signal-to-noise ratio (SNR) of user Bob1 and the instantaneous signal-to-interference-plus-noise ratio (SINR) of user Bob2 can be expressed as

$$
\gamma_{B 1}=\rho a_{1}\left|h_{B 1}\right|^{2} d_{B 1}^{-\alpha_{B 1}},
$$

and

$$
\gamma_{B 2}=\frac{a_{2}\left|\hat{h}_{B 2}\right|^{2} d_{1}^{-\alpha_{1}} d_{B 2}^{-\alpha_{B 2}}}{a_{1}\left|\hat{h}_{B 2}\right|^{2} d_{1}^{-\alpha_{1}} d_{B 2}^{-\alpha_{B 2}}+\frac{1}{\rho}},
$$

respectively, where $\rho$ denotes the transmit SNR, $\hat{h}_{B 2}=$ $\sum_{n=1}^{N}\left|g_{2, n}\right|\left|h_{n}\right|$ denotes the equivalent channel of BS-RISBob2 links.

The phase shifts are designed for user Bob2, hence the effective channel gain for Eve cannot be evaluated. In this paper, we consider the worst-case scenario of the RIS-aided NOMA network, in which all of the BS-RIS-Eve signals are co-phased. Therefore, the equivalent channel of Eve is similar to user Bob2's, which can be expressed as $\hat{h}_{E}=\sum_{n=1}^{N}\left|g_{E, n}\right|\left|h_{n}\right|$. Therefore, the instantaneous SNR of detecting the information of user Bob1 and user Bob2 can be expressed as:

$$
\gamma_{E_{i}}=\rho_{e} a_{i}\left|\hat{h}_{E}\right|^{2} d_{1}^{-\alpha_{1}} d_{E}^{-\alpha_{E}},
$$

where $i \in\{1,2\}$ and $\rho_{e}$ is the transmit SNR to the Eve. The cumulative distribution function (CDF) of $\gamma_{B 1}$ is given by

$$
F_{\gamma_{B 1}}(x)=1-e^{-\frac{x}{a_{1} \rho d_{B_{1}}^{-\alpha_{B 1}}}},
$$

Lemma 1. Recall that the fading parameters of the elements in $\mathbf{h}$ and $\mathbf{g}_{B 2}$ are $m_{1}$ and $m_{2}$, respectively. The CDF of $\gamma_{B 2}$ in the low-SNR regimes and the high-SNR regimes (when $m_{1} \neq m_{2}$ ) are given by

$$
F_{\gamma_{B 2}}(x)=1-Q_{\frac{1}{2}}\left(\sqrt{\lambda}, \sqrt{\frac{x}{N(1-\epsilon) \rho\left(a_{2}-a_{1} x\right) L}}\right),
$$

and

$$
F_{\gamma_{B 2}}^{0_{+}}(x)=\partial \gamma\left(2 m_{s} N, \frac{2 \sqrt{m_{s} m_{l} x}}{\sqrt{\rho\left(a_{2}-a_{1} x\right) L}}\right),
$$

respectively, where

$$
\begin{gathered}
\epsilon=\frac{1}{m_{1} m_{2}}\left(\frac{\Gamma\left(m_{1}+\frac{1}{2}\right)}{\Gamma\left(m_{1}\right)}\right)^{2}\left(\frac{\Gamma\left(m_{2}+\frac{1}{2}\right)}{\Gamma\left(m_{2}\right)}\right)^{2}, \\
\partial=\frac{m^{N}\left(4 m_{s} m_{l}\right)^{-m_{s} N}}{\Gamma\left(2 m_{s} N\right)},
\end{gathered}
$$

with

$$
m=\frac{\sqrt{\pi} 4^{m_{s}-m_{l}+1}\left(m_{s} m_{l}\right)_{s}^{m} \Gamma\left(2 m_{s}\right) \Gamma\left(2 m_{l}-2 m_{s}\right)}{\Gamma\left(m_{s}\right) \Gamma\left(m_{l}\right) \Gamma\left(m_{l}-m_{s}+\frac{1}{2}\right)},
$$

$Q_{\alpha}(\cdot, \cdot)$ is the Marcum $Q$-function, $L=d_{1}^{-\alpha_{1}} d_{B 2}^{-\alpha_{B 2}}, m_{l}=$ $\max \left(m_{1}, m_{2}\right), m_{s}=\min \left(m_{1}, m_{2}\right), \lambda=\frac{N \epsilon}{1-\epsilon}, \Gamma(\cdot)$ denotes the Gamma function and $\gamma(\cdot, \cdot)$ is the lower incomplete Gamma function.

Proof. Please refer to Appendix A.

Lemma 2. Denote that $Z=\sum_{n=1}^{N}\left|g_{E, n}\right|\left|h_{n}\right|$. The expectation 
of $Z^{2}$ is given by

$$
\mu=a N \omega^{N} d^{-a N} k_{1}^{N} \mho
$$

where $a=2 m_{c}, \quad b=m_{c}-m_{d}+\frac{1}{2}, c=m_{c}+m_{d}+$ $\frac{1}{2}, \quad d=2 \sqrt{m_{c} m_{d}}, \omega=\frac{\sqrt{\pi} 4^{m_{c}-m_{d}+1}\left(m_{c} m_{d}\right)^{m_{c}} \Gamma\left(2 m_{c}\right) \Gamma\left(2 m_{d}\right)}{\Gamma\left(m_{c}\right) \Gamma\left(m_{d}\right) \Gamma\left(m_{c}+m_{d}+\frac{1}{2}\right)}$ $m_{c}=\min \left(m_{1}, m_{3}\right), m_{d}=\max \left(m_{1}, m_{3}\right)$ and $\mho=\mho_{1}+$ $\mho_{2}-\mho_{3}+\mho_{4}$ with $\mho_{1}=\frac{a N+1}{d^{2}}, \mho_{2}=\frac{4 a b^{2} k_{2}^{2}}{c^{2} d^{2} k_{1}^{2}}(N-1)$, $\mho_{3}=\frac{4 a b N k_{2}}{c d^{2} k_{1}}$ and $\mho_{4}=\frac{4(a+1)\left(b^{2}+b\right)}{\left(c^{2}+c\right) d^{2}} k_{3}-\frac{4 b}{c d^{2}} k_{2}$. Furthermore, $k_{1}={ }_{2} F_{1}(a, b ; c ;-1), k_{2}={ }_{2} F_{1}(a+1, b+1 ; c+1 ;-1)$ and $k_{3}={ }_{2} F_{1}(a+2, b+2 ; c+2 ;-1)$ are the Gauss hypergeometric function [21, eq. (9.100)].

Proof. Please refer to Appendix B.

Remark 1. The use of moments results in (16) is accurate for the global CSIs. Hence, from (16), we can obtain that the expectation of channel gain for the reflected links is determined by the number of RISs and the Nakagami-m fading parameters.

\section{B. Secrecy Outage Probability}

In the proposed network, the capacity of LU is given by $C_{B_{i}}=\log \left(1+\gamma_{B_{i}}\right)$, while the capacity of the Eve's channel for the $i$-th user is quantified by $C_{E_{i}}=\log \left(1+\gamma_{E_{i}}\right)$. As such, the secrecy rate of the $i$-th user can be expressed as

$$
C_{i}=\left[C_{B_{i}}-C_{E_{i}}\right]^{+}
$$

where $[x]^{+}=\max \{x, 0\}$.

1) SOP analysis: we assumed that the targed rate is $R_{i}$. The SOP of the $i$-th user can be expressed as

$$
\begin{aligned}
P_{i}\left(R_{i}\right) & =\mathbb{P}\left(C_{i}<R_{i}\right) \\
& =\mathbb{P}\left(\log _{2}\left(\frac{1+\gamma_{B i}}{1+\gamma_{E i}}\right)<R_{i}\right) \\
& =\mathbb{P}\left(\gamma_{B i}<2^{R_{i}}\left(1+\gamma_{E i}\right)-1\right),
\end{aligned}
$$

Then we derive the SOP of user Bob1 and user Bob2 in the following theorems.

Theorem 1. In the considered RIS-aided NOMA network, the SOP of user Bobl is given by

$$
P_{1}\left(R_{1}\right) \approx 1-e^{\frac{-y_{1}}{a_{1} \rho d_{B_{1}}^{-\alpha_{1}} B_{1}}},
$$

where $y_{1}=2^{R_{1}}\left(1+a_{1} \rho_{e} \mu d_{1}^{-\alpha_{1}} d_{E}^{-\alpha_{E}}\right)-1$.

Proof. Based on (9) and (18), we have

$$
\begin{aligned}
P_{1}\left(R_{1}\right) & =\mathbb{P}\left(\gamma_{B_{1}}<2^{R_{1}}\left(1+\gamma_{E_{1}}\right)-1\right) \\
& \approx \mathbb{P}\left(\gamma_{B_{1}}<2^{R_{1}}\left(1+a_{1} \rho_{e} \mu d_{1}^{-\alpha_{1}} d_{E}^{-\alpha_{E}}\right)-1\right) \\
& =F_{\gamma_{B 1}}\left(y_{1}\right) .
\end{aligned}
$$

Then, by substituting (10) and (16) into (20), in the case of $m_{c}=\min \left(m_{1}, m_{3}\right)$ and $m_{d}=\max \left(m_{1}, m_{3}\right)$, we can obtain (19) after some mathematical manipulations. This completes the proof.
Theorem 2. The SOP of user Bob2 in the low-SNR regimes and high-SNR regimes (when $m_{1} \neq m_{2}$ ) are given by

$$
P_{2}^{l}\left(R_{2}\right) \approx e^{-\frac{\lambda}{2}} \sum_{k=0}^{\infty} \frac{\lambda^{k} \gamma\left(k+\frac{1}{2}, \frac{y_{l}}{2}\right)}{k ! 2^{k} \Gamma\left(k+\frac{1}{2}\right)}
$$

and

$$
P_{2}^{h}\left(R_{2}\right) \approx \partial \gamma\left(2 m_{s} N, 2 \sqrt{m_{s} m_{l}} y_{h}\right),
$$

respectively, where

$$
\begin{gathered}
y_{l}=\frac{y_{2}}{N(1-\epsilon) \rho\left(a_{2}-a_{1} y_{2}\right) d_{1}^{-\alpha_{1}} d_{B 2}^{-\alpha_{B 2}}}, \\
y_{h}=\frac{\sqrt{y_{2}}}{\sqrt{\rho\left(a_{2}-a_{1} y_{2}\right) d_{1}^{-\alpha_{1}} d_{B 2}^{-\alpha_{B 2}}}},
\end{gathered}
$$

and

$$
y_{2}=2^{R_{2}}\left(1+a_{2} \mu \rho_{e} d_{1}^{-\alpha_{1}} d_{E}^{-\alpha_{E}}\right)-1 .
$$

Proof. Based on (9), (18) and Lemma 2, the SOP of user Bob2 in the low-SNR and high-SNR regimes can be derived as

$$
P_{2}^{l}\left(R_{2}\right) \approx F_{\gamma_{B 2}}\left(y_{2}\right)
$$

and

$$
P_{2}^{h}\left(R_{2}\right) \approx F_{\gamma_{B 2}}^{0_{+}}\left(y_{2}\right)
$$

respectively. Then, by substituting (11) and (16) into (26), substituting (12) and (16) into (27), (21) and (22) can be obtained. This completes the proof.

Proposition 1. Both the SOP of user Bobl and the SOP of user Bob2 are 1 when the number of RISs is sufficiently large.

Proof. By substituting $N \rightarrow \infty$ into (16), we have

$$
\mu \approx a^{2} N^{2}\left(\frac{\omega k_{1}}{d}\right)^{N}\left(1-\frac{2 b k_{2}}{c k_{1}}\right)^{2}
$$

Since $c=1+a-b, k_{1}$ can be rewritten as $k_{1}=$ $\frac{\Gamma\left(m_{c}+m_{d}+\frac{1}{2}\right) \Gamma\left(1+m_{c}\right)}{\Gamma\left(1+2 m_{c}\right) \Gamma\left(m_{d}+\frac{1}{2}\right)}$. Let $\mu_{1}=\frac{\omega k_{1}}{d}$, then by substituting $k_{1}$ into $\mu_{1}$, we have

$$
\mu_{1}=\frac{\sqrt{\pi} 4^{m_{c}-m_{d}+1}\left(m_{c} m_{d}\right)^{m_{c}} \Gamma\left(2 m_{c}\right) \Gamma\left(2 m_{d}\right) \Gamma\left(1+m_{c}\right)}{\Gamma\left(m_{c}\right) 2 \sqrt{m_{c} m_{d}} \Gamma\left(1+2 m_{c}\right) \Gamma\left(m_{d}+\frac{1}{2}\right)} .
$$

Due to $\Gamma(1+x)=x \Gamma(x)$ and $\Gamma(x) \Gamma\left(x+\frac{1}{2}\right)=2^{1-2 x} \sqrt{\pi} \Gamma(2 x)$, (29) can be rewritten as

$$
\mu_{1}=2^{2 m_{c}-1}\left(m_{c} m_{d}\right)^{m_{c}-\frac{1}{2}} \Gamma\left(m_{c}\right) \Gamma\left(m_{d}\right) .
$$

Since $m_{d}>m_{c} \geq \frac{1}{2}$, we have $\mu_{1}>1$. Then by substituting (30) into (28), when $N \rightarrow \infty$, we have $\mu \rightarrow \infty$. By substituting $\mu$ in to Theorem 1 and Theorem 2, in the case of $N \rightarrow \infty$, we have $P_{1}\left(R_{1}\right)=P_{2}\left(R_{2}\right)=1$. This completes the proof.

\section{Asymptotic SOP and Secrecy Diversity Order Analysis}

In order to derive the secrecy diversity order to gain further insights into the network's operation in the high-SNR regimes, the asymptotic behavior is analyzed. Again, as the worst-case 
scenario, we assume that the Eve have a powerful detection capability, and all of the reflected signals are co-phased. Without loss of generality, it is assumed that the transmit SNR for the paired NOMA users is sufficiently high (i.e., $\rho \rightarrow \infty$ ), and the SNR of the BS-RIS-Eve links is set to arbitrary values. The secrecy diversity order can be defined as follows:

$$
d_{s}=-\lim _{\rho \rightarrow \infty} \frac{\log P^{\infty}}{\log \rho},
$$

where $P^{\infty}$ is the asymptotic SOP.

Corollary 1. The asymptotic SOP of user Bobl is given by

$$
P_{1}^{\infty}\left(R_{1}\right)=\frac{y_{1}}{a_{1} \rho d_{B_{1}}^{-\alpha_{B_{1}}}} .
$$

Proof. By expanding the exponential function in (19) and extracting the leading-order term, (32) is obtained. This completes the proof.

Remark 2. Upon substituting (32) into (31), the secrecy diversity order of user Bobl is 1.

Proposition 2. The floor of $P_{1}\left(R_{1}\right)$ in the case of $\rho_{e}=\rho$ is given by

$$
P_{1, \infty}^{\infty}\left(R_{1}\right)=\frac{2^{R_{1}} \mu d_{1}^{-\alpha_{1}} d_{E}^{-\alpha_{E}}}{d_{B_{1}}^{-\alpha_{B_{1}}}} .
$$

Proof. By Substituting $\rho_{e}=\rho$ in (32), after some mathematical manipulations, (33) can be obtained. This completes the proof.

Corollary 2. The asymptotic SOP of user Bob2 is given by

$$
P_{2}^{\infty}\left(R_{2}\right)=\frac{m^{N} y_{h}^{2 m_{s} N}}{\Gamma\left(2 m_{s} N+1\right)} .
$$

Proof. Based on Theorem 2, we have the SOP of user Bob2 in the high-SNR regimes. Then, by using the expansions of the lower incomplete Gamma function [21, eq. (8.354.1)], (22) can be represented as

$$
P_{2}^{\infty}\left(R_{2}\right)=\nsim \sum_{k=0}^{\infty} \frac{(-1)^{k}\left(2 \sqrt{m_{s} m_{l}} y_{h}\right)^{2 m_{s} N+k}}{k !\left(2 m_{s} N+k\right)} .
$$

By extracting the leading-order term in (35), (34) can be obtained. This completes the proof.

Remark 3. Upon substituting (34) into (31), the secrecy diversity order of user Bob2 is $m_{s} N$.

Remark 4. The secrecy diversity order of user Bobl is not affected by the number of RISs and Nakagami-m fading parameters. On the contrary, the secrecy diversity order of user Bob2 is affected by the number of RISs and Nakagami-m fading parameters.

\section{NUMERICAL RESUlTS}

In this section, our numerical results are presented for characterizing the performance of the considered network. Meanwhile, Monte-Carlo simulations are conducted to verify the accuracy. It is assumed that the power allocation coefficients

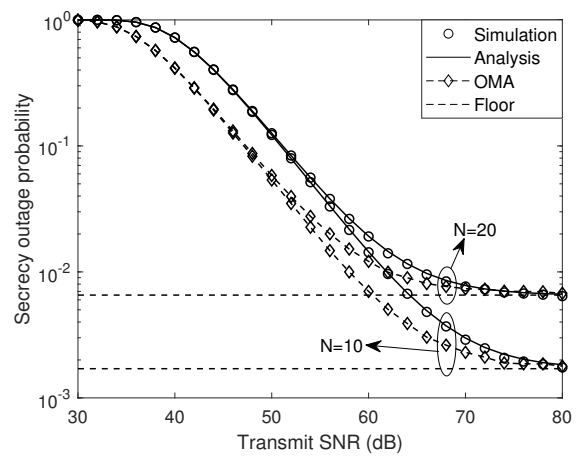

Fig. 2: SOPs for user Bob1 versus the transmit SNR. The analytical results and the floor are calculated from (19) and (33).

of NOMA are $a_{1}=0.2, a_{2}=0.8$, respectively. In addition, the amplitude reflection coefficients of RISs are set to 1 . The fading parameters are set to $m_{1}=3, m_{2}=m_{3}=1$. The length of the BS to RIS is set to $d_{1}=100 \mathrm{~m}$. The length of the RIS to user Bob2 and Eve are set to $d_{B 2}=10 \mathrm{~m}$ and $d_{E}=50 \mathrm{~m}$, and that of the BS-user Bob1 link is set to $d_{B 1}=20 \mathrm{~m}$. The path loss exponents of the reflected links (i.e., BS-RIS, RISBob2 and RIS-Eve) and the direct BS-Bob1 link are set to $\alpha_{1}=\alpha_{B 2}=\alpha_{E}=2.5$ and $\alpha_{B 1}=3.5$, respectively, unless otherwise stated. For comparisons, we regard the RIS-aided OMA network as the benchmark. Specifically, the RISs are employed for providing access service to user Bob2 as well as Eve to communicate with the BS.

Fig. 2 plots the SOP of user Bob1 versus the transmit SNR for different number of RISs. It confirms the close agreement between the simulation and analytical results. A specific observation is that the SOP of user Bob1 reduces as reducing the number of RISs. That is because the number of RISs has no effect on the channel gain of user Bob1, whereas, the channel gain of Eve increases as the number of RISs increases. As a benchmark, the SOP curves for the RIS-aided OMA network are plotted for comparison. We observe that for user Bob1 in the RIS-aided OMA network has better performance than that in the RIS-aided NOMA network in the high-SNR regimes. It is because that the transmit power allocated to user Bob1 in the NOMA network is lower than that in the OMA network due to the influence of the power allocation factor. As the transmit SNR increases, we find that the SOP of user Bob1 tends to a constant, which is consistent with Proposition 2.

Fig. 3 plots the SOP of user Bob2 versus the transmit SNR. We observe that, since the use of central limit theorem (CLT) in the channel statistics for user Bob2, the analytical results are accurate in the low-SNR regimes, but inaccurate in the highSNR regimes. As a benchmark, the SOP curves for the RISaided OMA network are plotted for comparison. We observe that the performance for user Bob2 in the RIS-aided NOMA network has superior performance than that in the RIS-aided OMA network. It is because the transmit power allocated to user Bob2 in the NOMA network is higher than that in the OMA network due to the influence of the power allocation 


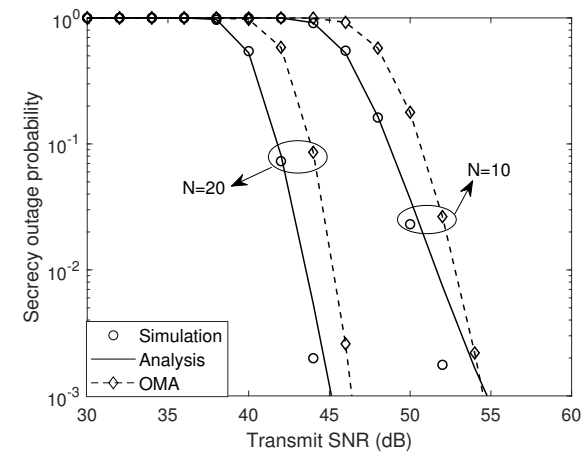

Fig. 3: SOPs for user Bob2 versus the transmit SNR. The analytical results are calculated from (21).

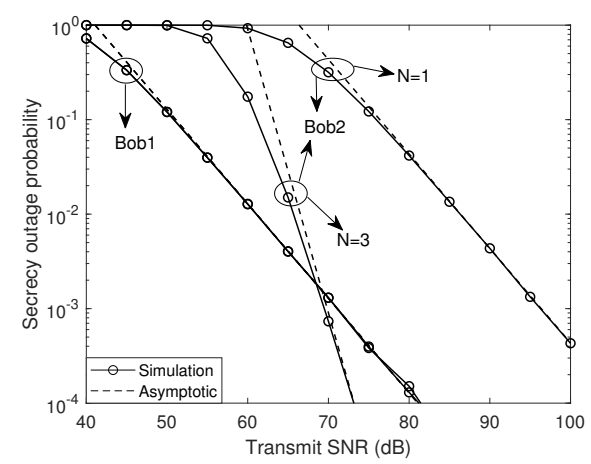

Fig. 4: Asymptotic results of SOP versus the transmit power in the case of $\rho_{e}=10 \mathrm{~dB}$. The asymptotic results are calculated from (32) and (34).

factor.

Since the SOP of uer Bob2 in the high-SNR regimes is not accurate in Fig. 3, we further plot the high-SNR asymptotic curves in the cases of $N=1$ and $N=3$ in Fig. 4 . We observe that the SOPs of user Bob1 and user Bob2 gradually approach their respective asymptotic curves, which validates our analysis. Furthermore, we also observe that, in the cases of $N=1$ and $N=3$, the secrecy diversity orders of user Bob1 are both 1 and the secrecy diversity orders of user Bob2 are 1 and 3, respectively, which is consistent with Remark 2 and Remark 3.

In Fig. 5, the SOP curves versus the number of RISs are depicted. We observe that, on the one hand, since we have global CSI for user Bob1, the SOP of user Bob1 is accurate. On the other hand, the SOP of user Bob2 is accurate in the lowSNR regimes. However, the SOP of user Bob2 is not accurate in the high-SNR regimes, which results from the use of the CLTbased channel statistics of user Bob2. We also observe that the SOP of user Bob1 increases as the number of RISs increases since the ER of user Bob1 is not affected by the number of RISs. On the contrary, the SOP of user Bob2 decreases as the number of RISs increases since the RIS transmission for Eve experience more severe path loss then that for user Bob2.

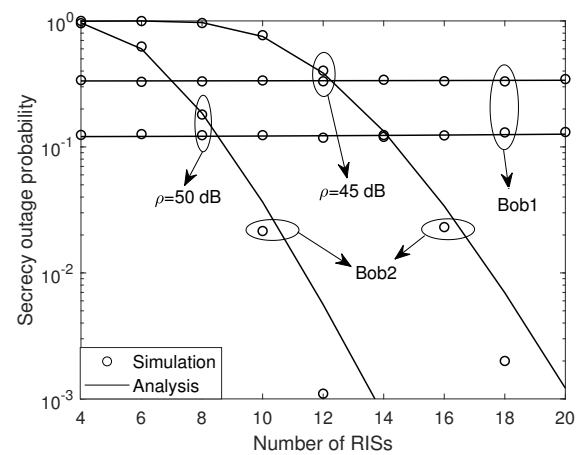

Fig. 5: SOPs versus the number of RISs. The analytical results are calculated from (19) and (21).

\section{CONCLUSiONS}

In this paper, the secrecy performance of the RIS-aided NOMA network was studied. Specifically, we first derived the new channel gain for the reflected links. Then, based on the new channel statistics, the closed-form results of the SOP were derived. Numerical results were presented for validating our results. Furthermore, secrecy diversity orders have been obtained for further insights. An important direction is that the presence of the direct link between the BS and the cell-edge user as well as Eve for the RIS-aided NOMA network is worthy of investigation for the future work.

\section{Appendix A: Proof of Lemma 1}

Firstly, according to [12], the CDF of $X=\frac{\left(\sum_{n=1}^{N}\left|g_{2, n}\right|\left|h_{n}\right|\right)^{2}}{N(1-\epsilon)}$ in the low-SNR regimes is given by

$$
F_{X}(x)=1-Q_{\frac{1}{2}}(\sqrt{\lambda}, \sqrt{x})
$$

Hence, the CDF of $\gamma_{B 2}$ in the low-SNR regimes can be derived as

$$
\begin{aligned}
F_{\gamma_{B 2}}(x) & =\mathbb{P}\left(\gamma_{B 2}<x\right) \\
& =F_{X}\left(\frac{x}{N(1-\epsilon) \rho\left(a_{2}-a_{1} x\right) d_{1}^{-\alpha_{1}} d_{B 2}^{-\alpha_{B 2}}}\right) .
\end{aligned}
$$

Then, according to [12], the CDF of $Y=\sum_{n=1}^{N}\left|g_{2, n}\right|\left|h_{n}\right|$ in the high-SNR regimes is given by

$$
F_{Y}(y)=\partial \gamma\left(2 m_{s} N, 2 \sqrt{m_{s} m_{l}} y\right) .
$$

Therefore, the CDF of $\gamma_{B 2}$ in the high-SNR regimes can be derived as

$$
\begin{aligned}
F_{\gamma_{B 2}}^{0_{+}}(x) & =\mathbb{P}\left(\gamma_{B 2}<x\right) \\
& =F_{Y}\left(\sqrt{\frac{x}{\rho\left(a_{2}-a_{1} x\right) d_{1}^{-\alpha_{1}} d_{B 2}^{-\alpha_{B 2}}}}\right) .
\end{aligned}
$$

By substituting (A.1) into (A.2), and substituting (A.3) into (A.4), (11) and (12) can be obtained. This completes the proof. 


\section{APPENDIX B: PROOF OF LEMMA 2}

Denote that $z_{n}=\left|g_{E, n}\right|\left|h_{n}\right|$, and $f_{z_{n}}$ is the probability density function (PDF) of $z_{n}$, according to [12], the Laplace transform of $f_{z_{n}}$ is given by

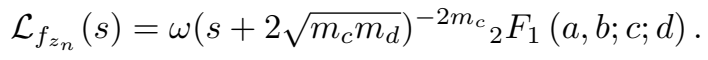

Denote that $f_{Z}$ is the PDF of $Z$. Therefore, the Laplace transform of $f_{Z}$ is given by

$$
\mathcal{L}_{f_{Z}}(s)=\underbrace{\omega^{N}(s+d)^{-a N}}_{f(s)} \underbrace{\left({ }_{2} F_{1}\left(a, b ; c ; \frac{s-d}{s+d}\right)\right)^{N}}_{g(s)} .
$$

According to the relationship between Laplace transform and moments, we have

$$
\mathbb{E}\left(Z^{2}\right)=\mathcal{L}_{f_{Z}}^{\prime \prime}(0)
$$

From (B.2), we have

$$
\mathcal{L}_{f_{Z}}^{\prime}(s)=J_{1}(s)+J_{2}(s) J_{4}(s)
$$

where

$$
\begin{gathered}
J_{1}(s)=-a N \omega^{N}(s+d)^{-a N-1} g(s), \\
J_{2}(s)=f(s) \underbrace{N\left({ }_{2} F_{1}\left(a, b ; c ; \frac{s-d}{s+d}\right)\right)^{N-1}}_{J_{3}(s)},
\end{gathered}
$$

and

$$
J_{4}(s)=\frac{a b}{c}{ }_{2} F_{1}\left(a+1, b+1 ; c+1 ; \frac{s-d}{s+d}\right) \frac{2 d}{(s+d)^{2}} .
$$

Furthermore, we have

$$
\mathcal{L}_{f_{Z}}^{\prime \prime}(0)=J_{1}^{\prime}(0)+J_{2}^{\prime}(0) J_{4}(0)+J_{2}(0) J_{4}^{\prime}(0),
$$

where

$$
\begin{gathered}
J_{2}(0)=\omega^{N} d^{-a N} N k_{1}^{N}, \\
J_{4}(0)=\frac{2 a b}{c d} k_{2}, \\
J_{1}^{\prime}(0)=a N \omega^{N} d^{-a N-2} k_{1}^{N-1}\left((a N+1) k_{1}-\frac{2 a b}{c} N k_{2}\right), \\
J_{2}^{\prime}(0)=\omega^{N} d^{-a N-1} N k_{1}^{N-2}\left(\frac{2 a b}{c}(N-1) k_{2}-a N k_{1}\right), \\
J_{4}^{\prime}(0)=\frac{4 a(a+1) b(b+1)}{c(c+1) d^{2}} k_{3}-\frac{4 a b}{c d^{2}} k_{2} .
\end{gathered}
$$

Then, by substituting (B.9)-(B.13) into (B.8), and after some further mathematical manipulations, (16) can be obtained. This completes the proof.

\section{ACKNOWLEDGMENT}

This work was supported in part by the National Science Foundation of China under Grant 61571401, in part by the National Science Foundation for Young Scientists of China under Grant 61901416.

\section{REFERENCES}

[1] Q. Wu and R. Zhang, "Intelligent reflecting surface enhanced wireless network via joint active and passive beamforming," IEEE Trans. Wireless Commun., vol. 18, no. 11, pp. 5394-5409, Aug. 2019.

[2] R. Alghamdi, R. Alhadrami, D. Alhothali, H. Almorad, A. Faisal, S. Helal, R. Shalabi, R. Asfour, N. Hammad, A. Shams, N. Saeed, H. Dahrouj, T. Y. Al-Naffouri, and M. S. Alouini, "Intelligent surfaces for $6 \mathrm{G}$ wireless networks: A survey of optimization and performance analysis techniques," IEEE Access, vol. 8, pp. 202 795-202 818, Oct. 2020.

[3] C. Huang, A. Zappone, G. C. Alexandropoulos, M. Debbah, and C. Yuen, "Reconfigurable intelligent surfaces for energy efficiency in wireless communication," IEEE Trans. Wireless Commun., vol. 18, no. 8, pp. 4157-4170, Jun. 2019.

[4] M. Di Renzo, M. Debbah, D.-T. Phan-Huy, A. Zappone, M.-S. Alouini, C. Yuen, V. Sciancalepore, G. C. Alexandropoulos, J. Hoydis, H. Gacanin et al., "Smart radio environments empowered by reconfigurable AI metasurfaces: An idea whose time has come," EURASIP J. Wireless Commun. Netw., vol. 2019, no. 1, pp. 1-20, May 2019.

[5] M. Di Renzo and J. Song, "Reflection probability in wireless networks with metasurface-coated environmental objects: An approach based on random spatial processes," EURASIP J. Wireless Commun. Netw., vol. 2019, no. 1, p. 99, Apr. 2019.

[6] T. Hou, Y. Liu, Z. Song, X. Sun, and Y. Chen, "Mimo-noma networks relying on reconfigurable intelligent surface: A signal cancellation-based design,” IEEE Trans. Wireless Commun., vol. 68, no. 11, pp. 6932-6944, Nov. 2020.

[7] M. Di Renzo, A. Zappone, M. Debbah, M.-S. Alouini, C. Yuen, J. de Rosny, and S. Tretyakov, "Smart radio environments empowered by reconfigurable intelligent surfaces: How it works, state of research, and the road ahead," IEEE J. Sel. Areas Commun., vol. 38, no. 11, pp. 2450-2525, Nov. 2020.

[8] Y. Liu, Z. Qin, M. Elkashlan, Z. Ding, A. Nallanathan, and L. Hanzo, "Non-orthogonal multiple access for 5G and beyond," IEEE Proc., vol. 105, no. 12, pp. 2347-2381, Dec. 2017.

[9] Z. Ding, Y. Liu, J. Choi, Q. Sun, M. Elkashlan, I. Chih-Lin, and H. V. Poor, "Application of non-orthogonal multiple access in LTE and 5G networks," IEEE Commun. Mag., vol. 55, no. 2, pp. 185-191, Feb. 2017.

[10] Z. Ding, P. Fan, and H. V. Poor, "Impact of user pairing on 5G nonorthogonal multiple-access downlink transmissions," IEEE Trans. Veh. Technol., vol. 65, no. 8, pp. 6010-6023, Sep. 2015.

[11] T. Hou, Y. Liu, Z. Song, X. Sun, Y. Chen, and L. Hanzo, "Reconfigurable intelligent surface aided NOMA networks," IEEE J. Sel. Areas Commun., pp. 1-1, Jul. 2020.

[12] Y. Cheng, K. H. Li, Y. Liu, K. C. Teh, and H. V. Poor, "Downlink and uplink intelligent reflecting surface aided networks: NOMA and OMA," IEEE Trans. Wireless Commun., Early Access, 2021.

[13] Z. Ding and H. V. Poor, "A simple design of IRS-NOMA transmission," IEEE Commun. Lett., vol. 24, no. 5, pp. 1119-1123, May 2020.

[14] A. Almohamad, A. M. Tahir, A. Al-Kababji, H. M. Furqan, T. Khattab, M. O. Hasna, and H. Arslan, "Smart and secure wireless communications via reflecting intelligent surfaces: A short survey," IEEE Open J. Commun. Soc., vol. 1, pp. 1442-1456, Sep. 2020.

[15] L. Yang, Y. Jinxia, W. Xie, M. Hasna, T. Tsiftsis, and M. Di Renzo, "Secrecy performance analysis of RIS-Aided wireless communication systems," IEEE Trans. Veh. Technol., pp. 1-1, Jul. 2020.

[16] M. Cui, G. Zhang, and R. Zhang, "Secure wireless communication via intelligent reflecting surface," IEEE Wireless Commun. Lett., vol. 8, no. 5, pp. 1410-1414, May 2019.

[17] X. Guan, Q. Wu, and R. Zhang, "Intelligent reflecting surface assisted secrecy communication: Is artificial noise helpful or not?" IEEE Wireless Commun. Lett., vol. 9, no. 6, pp. 778-782, Jan. 2020.

[18] L. Dong and H.-M. Wang, "Secure MIMO transmission via intelligent reflecting surface," IEEE Wireless Commun. Lett., vol. 9, no. 6, pp. 787790, Jan. 2020.

[19] Z. Chu, W. Hao, P. Xiao, and J. Shi, "Intelligent reflecting surface aided multi-antenna secure transmission," IEEE Wireless Commun. Lett., vol. 9, no. 1, pp. 108-112, Sep. 2020.

[20] H. Shen, W. Xu, S. Gong, Z. He, and C. Zhao, "Secrecy rate maximization for intelligent reflecting surface assisted multi-antenna communications," IEEE Commun. Lett., vol. 23, no. 9, pp. 1488-1492, Jun. 2019.

[21] I. S. Gradshteyn and I. M. Ryzhik, Table of Integrals, Series, and Products, 7th ed. Amsterdam, The Netherlands: Elsevier, 2007. 


\section{EDAS Conference Manager on behalf of ieeeicc2021workshops-chairs}

To Jiankang Zhang

From:

EDAS Conference Manager<help@edas.info> on behalf of ieeeicc2021workshops-

chairs<ieeeicc2021workshops-chairs@edas.info>

To: Jiankang Zhang<jz09v@ecs.soton.ac.uk>

Time: 31 March 2021 (Wed) 02:45

Size: 165 KB

CAUTION: This e-mail originated outside the University of Southampton.

Dear Dr. Jiankang Zhang:

Congratulations again on the acceptance of your paper \#1570706998 ('Secrecy Performance Analysis for Reconfigurable Intelligent Surface aided NOMA Network') for WS10 ICC'21 Workshop - Intelli-Surfaces for presentation at IEEE ICC 2021-Workshops.

Accepted and presented papers will be published in the IEEE ICC 2021 Workshop Proceedings and submitted to IEEE Xplore ${ }^{\circledR}$ (subject to the fulfillment of conditions outlined below - see also full COMSOC policy at the end of the message).

\section{PLEASE READ ALL OF THE IMPORTANT INFORMATION BELOW THOROUGHLY.}

\section{Registration}

In order to be published in the IEEE ICC 2021 Proceedings and IEEE Xplore $\AA_{\text {, an author }}$ (including students) of an accepted paper is required to register for the conference at the Presenting Author rate (which is discounted for IEEE and ComSoc members) and the paper must be presented (as a prerecorded video) by an author or authors named on the paper. Non-refundable registration fees must be paid prior to uploading the final IEEE formatted, publication-ready version of the paper.

For authors with multiple accepted papers, one Presenting Author registration is valid for up to 3 (THREE) papers in a given workshop. Accepted and presented papers will be published in the IEEE ICC 2021 Workshop Proceedings and in IEEE Xplore ${ }^{\circledR}$. Although the registration confirmation code enables submission of up to THREE papers, it can only be used once, by you, to create an author account for your submissions. Please do not share your registration confirmation code with anyone else. Registration is open at https://eur03.safelinks.protection.outlook.com/?url=https\%3A\%2F\%2Ficc2021.ieeeicc.org\%2Fregistration\&data =04\%7C01\%7Cjz1 n08\%40soton.ac.uk\%7Cdeec0cdd1b784f65c76cC

\section{Paper Revision and Final Manuscript Upload}

Each accepted paper is limited to 6 pages (or no more than 7 pages with USD 100 over-length charge for the extra page). You are strongly recommended to revise your paper in consideration of the reviewers' comments. Your Workshop Organizers may contact you if they are not satisfied with your final version of your paper. Please note that the paper title as well as the list and order of authors CANNOT be changed after the paper is accepted. Failure to abide by this policy may result in the removal of your paper from the final conference program.

The final manuscript is due by March 31, 2021, but you must be registered for IEEE ICC 2021 Workshops to upload your paper. Once you have registered, you will receive a confirmation code that you will use when you upload the paper on eXpress (NOT EDAS). Details on the 
upload process are posted in the "Information for Authors/Speakers" page of the IEEE ICC 2021 website at https://eur03.safelinks.protection.outlook.com/?url=https\%3A\%2F\%2Ficc2021.ieeeicc.org\%2F\&data $=04 \% 7$ C01\%7Cjz1n08\%40soton.ac.uk\%7Cdeec0cdd1b784f65c76c08d8f3e6a7s

\section{Presentation Recording}

IEEE ICC 2021 will be hosted entirely online. The authors of each accepted and registered paper are required to upload a pre-recorded video of their paper presentation. The video must have a duration of approximately 15 minutes and must be uploaded to EDAS no later than April 15, 2021. The video will be released during the conference and will be made available to registered attendees on-demand both during the conference and after it concludes.

Full instructions for recording and uploading the video can be found here: https://eur03.safelinks.protection.outlook.com/?url=https\%3A\%2F\%2Ficc2021.ieeeicc.org\%2Fauthor-video-upload-

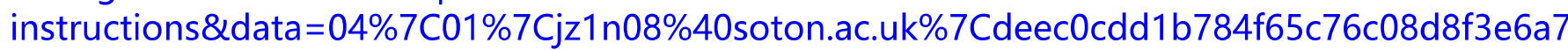

Failure to upload the video on time or to meet the technical and length requirements will result in the paper not being submitted to IEEE Xplore in accordance with the COMSOC noshow policy (see below).

Sessions and Interactivity

Your paper will be assigned to a session, which will be listed on your paper's EDAS page. The online platform will include features that will allow you to interact with other attendees and answer the questions they may have regarding your paper. More instructions on how to handle questions about your paper will be provided later.

\section{No-Show Policy}

The organizers of IEEE ICC 2021 as well as attendees expect an accepted paper to be presented at the conference by the author of that paper. As an online conference, this requirement is fulfilled by recording and uploading an acceptable video prior to the deadline. A paper that fails to meet this requirement will be removed from the final conference proceedings before uploading to IEEE Xplore ${ }^{\circledR}$. No refund will be made to authors of these papers. Please read carefully the full policy shown below under "IEEE COMSOC POLICY".

\section{IEEE COMSOC POLICY}

To be published in the IEEE ICC 2021 Conference Proceedings and to be eligible for publication in IEEE Xplore ${ }^{\circledR}$, an author of an accepted paper is required to register for the conference at the AUTHOR (member or non-member) rate and the paper must be presented by an author of that paper at the conference unless the TPC Chair grants permission for a substitute presenter arranged in advance of the event and who is qualified both to present and answer questions. Non-refundable registration fees must be paid prior to uploading the final IEEE formatted, publication-ready version of the paper. For authors with multiple accepted papers, one full registration is valid for up to 3 papers. Accepted and presented papers will be published in the IEEE ICC 2021 Conference Proceedings and submitted to IEEE Xplore ${ }^{\circledR}$ as well as other Abstracting and Indexing (A\&l) databases.

We look forward to welcoming you online in June 2021.

Thank you and best regards,

Alessio Zappone, Marco Di Renzo, Wei Xu, Chau Yuen, Mark Flanagan, Daniel Benevides da Costa

IEEE ICC 2021 Intelli-Surfaces Workshop Co-Chairs 